\title{
Effects of single-dose ketamine infusion on behavioral parameters and neuronal activation in the medial prefrontal cortex of juvenile rats exposed to prenatal stress
}

\author{
Elif Polat Çorumlu ${ }^{1}$ Osman Özcan Aydın ${ }^{1}$ Emine Gülhan Aydın², Emel Ulupınar ${ }^{1,3}$ \\ ${ }^{1}$ Department of Interdisciplinary Neuroscience, Institute of Health Sciences, Eskişehir Osmangazi University, Eskişehir, Turkey \\ ${ }^{2}$ Department of Pharmacology, Faculty of Medicine, Eskişehir Osmangazi University, Eskişehir, Turkey \\ ${ }^{3}$ Department of Anatomy, Faculty of Medicine, Eskişehir Osmangazi University, Eskişebir, Turkey
}

\begin{abstract}
Objectives: Subanesthetic dose of ketamine administration produces antidepressant-like response especially in treatmentresistant depression patients. Ketamine's rapid and sustained actions have also been demonstrated in normal or chronically stressed animals, but no experimental studies have examined its effects in prenatally stressed rodents. Therefore, aim of the present study was to investigate the behavioral and structural consequences of single-dose ketamine application in juvenile rats exposed to prenatal stress.

Methods: Prenatal stress protocol was applied by immobilization of pregnant rats during the last week of their gestation for 3 hours a day. While treatment group received a single-dose (10 mg/kg) of intraperitoneal ketamine injection, control and stress groups received same amount of saline injections at P38. After completion of behavioral tests (sucrose preference, modified grip and forced swim), animals were sacrificed via intracardiac perfusion. Then, immediate gene expression in the medial prefrontal cortex was evaluated by c-Fos immunohistochemistry.

Results: Although the active coping and depressive-like behavior of juvenile animals exposed to prenatal stress did not significantly change, ketamine application caused alterations in the sucrose preference pattern of animals and immobility time in the forced swim test. Two-way ANOVA test results showed significant differences among groups and a group $X$ gender interaction in the density of c-Fos expressing neurons present in the medial prefrontal cortex.

Conclusion: A single-dose ketamine treatment might differentially affect the depressive-like behaviors of juvenile animals exposed to prenatal stress and activate neurons in the medial prefrontal cortical region in a gender-dependent manner.
\end{abstract}

Keywords: c-Fos; depression; ketamine; prefrontal cortex; prenatal stress

Anatomy 2015;9(3):142-150 @2015 Turkish Society of Anatomy and Clinical Anatomy (TSACA)

\section{Introduction}

Major depressive disorder (MDD) is the leading cause of disability worldwide. ${ }^{[1]}$ This disease is characterized by loss of interest in daily activities, sadness, and feelings of tiredness, guilt or low self-worth. Since these symptoms disturb sleep, appetite and concentration skills of individuals, depression can be defined as a debilitating disor- der by its growing direct and indirect financial burden on health care spending. The estimated lifetime prevalence varies across the cultures from $3 \%$ to $16.9 \% .^{[2]}$

Currently, there are more than twenty different antidepressant medications targeting the monoamine systems. These drugs generally work by increasing the amount of serotonin or norepinephrine in the brain. While effective in most patients, sustained remission is 
achieved only in one-third of patients after treatment which puts them at greater risk of alcohol and drug abuse, hospitalization, and even suicide attempts. ${ }^{[3]}$ The full clinical benefits of conventional antidepressants take weeks to months start working. ${ }^{[4]}$ This time lag to therapeutic efficacy is a serious problem especially in considering the high suicide risk associated with MDD. For patients with severe depression or those having thoughts of self-harm, immediate relief is needed and may even be lifesaving. Until now, electro-convulsive therapy (ECT) has been the only proven method of treating severe and life threatening depression with minimal delay. However, this is an invasive method, in which electrical shock induces seizures in patients, and failed to gain a general acceptance. ${ }^{[5]}$ Therefore, there is urgent need of fast-acting, well-tolerated and more effective treatment method especially for patients at the high risk of suicide.

For over a decade, scientists have been investigating the role of other neurotransmitters and mechanisms in understanding the pathophysiology of treatment resistant MDD. Now, a growing body of research indicates there may be a new hope: a well-known general anesthetic drug ketamine. Ketamine is a non-competitive glutamate N-methyl-D-aspartate (NMDA) receptor antagonist and subanesthetic dose of this drug is associated with antidepressant effect in patients with MDD. Stimulation of glutamate transmission by ketamine results in BDNF release and activation of signaling pathways critically involved in the formation, maturation and function of new synapses. ${ }^{[6]}$ Unlike other antidepressants, ketamine has a very rapid and dramatic onset of antidepressant response. ${ }^{[7,8]}$ This feature can be extremely helpful in emergency rooms for curbing suicidal thoughts and making it a potential lifesaver. ${ }^{[9]}$

Effects of ketamine were tested in both patients with MDD and animal models of depression. ${ }^{[10,11]}$ In these basic and clinical studies, morphological and functional alterations have been shown in the brain regions that are critical for the regulation of mood and cognition, including the prefrontal cortex (PFC). ${ }^{[12]}$ Post mortem studies of patients with MDD revealed reduced number of synapses in the PFC. ${ }^{[13]}$ Stress exposure during prenatal or adult period, a well-known depression model in rodents, also causes deficits in medial PFC neurons. ${ }^{[1,15]}$ We have recently shown that in perinatally stressed animals, dendritic complexity and spine density of pyramidal neurons in the medial PFC display anxiety level- and sex-dependent reductions. ${ }^{[16]}$ Moreover, accumulating evidence suggests that a dysfunction in the glutamatergic system is evident in the prefrontal cortical neurons of MDD patients. ${ }^{[17]}$
Imaging studies showed that subanesthetic doses of ketamine increased cerebral blood flow and glucose metabolic rate especially in the thalamus, frontal and parietal cortical regions. ${ }^{[18]}$ It has been shown that reduced activity in the $\mathrm{PFC}$ region of depressed patients is normalized following ketamine treatment. ${ }^{[19]}$ In a series of preclinical studies, ketamine has been shown to induce synaptic signaling proteins and neuroplastic changes in the PFC of rats. ${ }^{[20-22]}$

Prolonged development of the PFC makes it susceptible to adverse conditions. Although recent welldesigned studies are beginning to yield important insights into the cellular and molecular mechanisms of ketamine's antidepressant action, the role of ketamine in juvenile animal models of depression has not been investigated yet. Therefore, in this study, by using behavioral and morphological analyses, the effect of single-dose ketamine application was investigated in juvenile rats exposed to prenatal stress.

\section{Materials and Methods \\ Stress protocol and animal treatment}

Sprague-Dawley rats were obtained from the breeding colony at the Eskişehir Osmangazi University Animal Care Facility. The laboratory was maintained under constant temperature $\left(21^{\circ} \mathrm{C}\right)$ and lighting $(12: 12 \mathrm{~h} \mathrm{light/dark}$ cycle) conditions. After overnight mating with breeder male rats, pregnancy was confirmed by the sperm positivity in vaginal smear and this particular day was assigned as embryonic day (E) 0. Pregnant rats were housed individually in transparent cages by giving free access to food and tap water. Prenatal stress protocol was applied by keeping the dams $(n=10)$ immobile in close-fitting wire mesh cylinders for $3 \mathrm{~h}$, between E14 and E21. In the treatment group, prenatally stressed animals received a single-dose of ketamine injection $(10 \mathrm{mg} / \mathrm{kg})$ intraperitoneally, at P38. Control and prenatal stress groups $(n=4$ for each gender in each group) received same amount of saline solution. Experimental procedures were performed in accordance with protocols approved by the Institutional Animal Usage Committee of Eskişehir Osmangazi University Faculty of Medicine (Protocol \#201/1).

\section{Sucrose preference test}

Animals were initially subjected to a palatable sucrose solution (1\%; Sigma) for 48 hours and then exposed to a water deprivation period for 4 hours. At the end of this period, they were exposed to two identical bottles, containing either water or sucrose solution for one hour. Sucrose and water consumption were determined by measuring the change in the volume of fluid consumed. 
The sucrose preference percentage (SPP) was calculated as follows: SPP $=$ [sucrose solution intake $(\mathrm{ml}) /$ (sucrose solution intake $(\mathrm{ml})+$ sterile water intake $(\mathrm{ml})] \times 100$. The cut off point for anhedonia, as an indicator of depressive symptom, is considered as under $0.65 .^{[23]}$

\section{Modified grip suspension test}

The endurance capacity and escape behavior of animals were determined by using an apparatus consisting of a string $(50 \mathrm{~cm}$ length and $3 \mathrm{~mm}$ in diameter) pulled straight between two vertical supports, $80 \mathrm{~cm}$ above the blanket-covered tabletop. ${ }^{[2]]}$ The front paws of the rats were placed on the horizontal string, at a point midway between the supports, and the latency before the animals fell from the string was recorded. In addition, escape behavior of an animal was rated by two blinded experimenters according to the following scale: $0=$ Fall off; 1 $=$ Hangs on string only by forepaws; 2 = As for 1 , but attempts to climb on string; 3 = Hangs onto string by two forepaws plus one or both hind paws; 4 = Hangs onto string by all fore and hind paws plus tail wrapped around string; 5 = Staying on the string for more than $150 \mathrm{~s}$ or escape after $50 \mathrm{~s} ; 6=$ Escape in between 41 and $50 \mathrm{~s} ; 7$ = Escape in between 31 and $40 \mathrm{~s} ; 8=$ Escape in between 21 and $30 \mathrm{~s} ; 9=$ Escape in between 11 and $20 \mathrm{~s}$; $10=$ Escape in the first $10 \mathrm{~s}$ of the test. The highest score for each rat was obtained from two trials with an interval of approximately 15-20 minutes.

\section{Forced swim test}

Forced swim test (FST) is a two-day procedure performed as described previously. ${ }^{[25]}$ On the first day, rats were placed in large inescapable Plexiglas cylinders $(30 \times$ $45 \mathrm{~cm}$ ) filled with $25^{\circ} \mathrm{C}$ water to a depth of $30 \mathrm{~cm}$, and let to swim for 15 minutes. On the second day, animals were forced to swim again under the same conditions, for 5 minutes. The cylinders were emptied and cleaned at the end of each swimming test. Behavior of animals during swimming period was videotaped and the immobility time was recorded. The duration of immobility was defined as the period when the animals made no escapeoriented movement, as a sign of depressive behavior.

\section{Tissue preparation and immunohistochemical analysis}

In order to estimate the level of immediate gene expression, c-Fos immunohistochemistry was used. Since forced swimming was also used to stimulate neuronal activity, animals were sacrificed immediately after the FST by cardiac perfusion under the halothane anesthesia. All animals were perfused with phosphate-buffered saline (PBS) followed by a fixative containing $4 \%$ paraformaldehyde in $0.1 \mathrm{M}$ phosphate buffer $(\mathrm{pH}=7.4)$. The brains were removed from the skulls and dissected into two hemispheres. Left hemispheres were left in the fixative solution for $24 \mathrm{~h}$ at $4^{\circ} \mathrm{C}$, followed by dehydration in graded alcohols, then embedded in paraffin. All tissue blocks were fixed and processed under the same conditions. The tissue was sectioned at 5$\mu \mathrm{m}$ thickness and every 20 th section was mounted on polyL-lysine coated slides. After deparaffinization and rehydration, sections were treated with $0.3 \% \mathrm{H}_{2} \mathrm{O}_{2}$ for $30 \mathrm{~min}$, washed in PBS, and then boiled in antigen retrieval solution, containing sodium citrate $(\mathrm{pH}=6.00)$ for $5 \mathrm{~min}$ in a microwave oven. Unspecific binding was suppressed in a blocking solution for $30 \mathrm{~min}$ at room temperature. The rabbit polyclonal antibody to c-Fos (Merck-Millipore, Darmstadt, Germany) was used at 1:200 dilutions. After overnight incubation at $4^{\circ} \mathrm{C}$, sections were rinsed with PBS and incubated in biotinylated anti-rabbit secondary antibody (1:100, Novostain Universal Detection Kit; Novocastra Laboratories, Leica Biosystems Newcastle Ltd., Newcastle, UK) for $30 \mathrm{~min}$ at room temperature. Bound antibody was detected by the ready-to-use streptavidin/peroxidase complex reagent (ABC-Elite Kit; Vector Laboratories, Burlingame, CA, USA), and diaminobenzidine $(\mathrm{DAB})$ mixture as chromogen. Finally sections were dehydrated in alcohol series, cleared in xylene, and coverslipped with permanent mounting medium.

\section{Estimation of neuronal density}

Quantitative analyses were done in sections obtained through the rostro-caudal extent, between Bregma 2.76 and 0.48 , according to Paxinos and Watson's stereotaxic rat atlas. ${ }^{[26]}$ Systematic random sampling method was applied in every step and each slide was identified with a code not revealed until the end of quantifications. Photographs of the medial prefrontal cortical regions were used to analyze the mean number of c-Fos (+) nuclei within a two-dimensional counting frame $(600 \mu \mathrm{m}$ $\times 600 \mu \mathrm{m}$ in size) by using a $40 \times$ objective. The size of the counting frame was set up to generate about 8-10 sampling sites on average, per tissue section. The counting frame locations were kept constant for each section, and the nuclear profiles were counted in every 20th section. Approximately 10 sections were used for quantitative analyses per animal.

\section{Statistical analysis}

The data presented in the text and tables represents mean \pm standard error of means (SEM). Normality distribution of data was assessed by the Shapiro-Wilk tests. Groups were compared using two-way ANOVA (group 


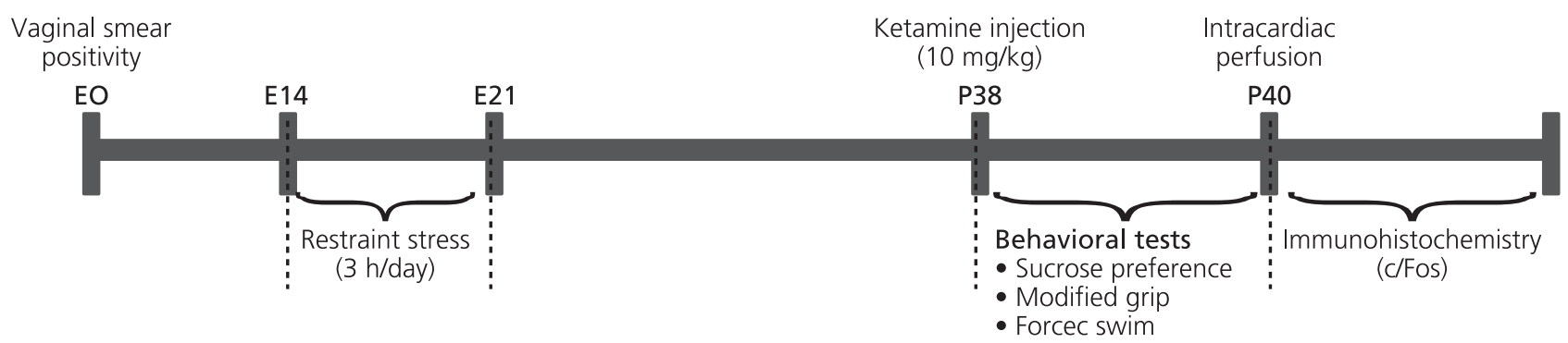

Figure 1. Time table of the experimental design.

$\times$ gender) followed by post-hoc pairwise Bonferroni multiple comparisons or Kruskal-Wallis test followed by Dunn's multiple comparisons test. Results were considered significant at a level of $\mathrm{p}<0.05$. All analyses were performed using SPSS Statistics Version 21 software.

\section{Results}

Prenatal stress exposure model has been successfully established and utilized as a depression model in our laboratory ${ }^{[24]}$ In the present study, we kept pregnant dams immobile for 3 hours daily during the last week of their gestation period, and then evaluated the behavioral consequences of prenatal stress on pups following ketamine injection (Figure 1). In animals exposed to prenatal stress, behavioral parameters related to human depression symptoms, such as loss of active coping, social withdrawal and inability to feel pleasure (anhedonia) can be evaluated under appropriate experimental conditions. ${ }^{[27]}$ For instance, anhedonia can be assessed by comparing the preference for sucrose over water in home cages of animals. In our study, mean sucrose consumption of prenatally stressed rats was comparable to those of unstressed controls. However, when we considered the individual differences, sucrose consumption rate was below the threshold level $(0.65 \%)$, indicating an anhedonic response, especially in males exposed to prenatal stress (Figure 2). Following ketamine injection, majority of both female and male animals preferred sucrose over water.

The performance of animals in the modified grip test remained consistent across different groups (Figure 3). Although grip test is more valuable for evaluation of the motor coordination ability, here, we have used this test to compare the active coping and endurance capacity of animals. In comparison to prenatally stressed males, average grip test score was increased after ketamine injection (8 versus 9), but their statistical comparisons did not reach to significance level.

Loss of coping is also seen as a sign of learned helplessness and it can be assessed by inescapable stress in the forced swim test. ${ }^{[28]}$ In this test, too, females displayed sim-

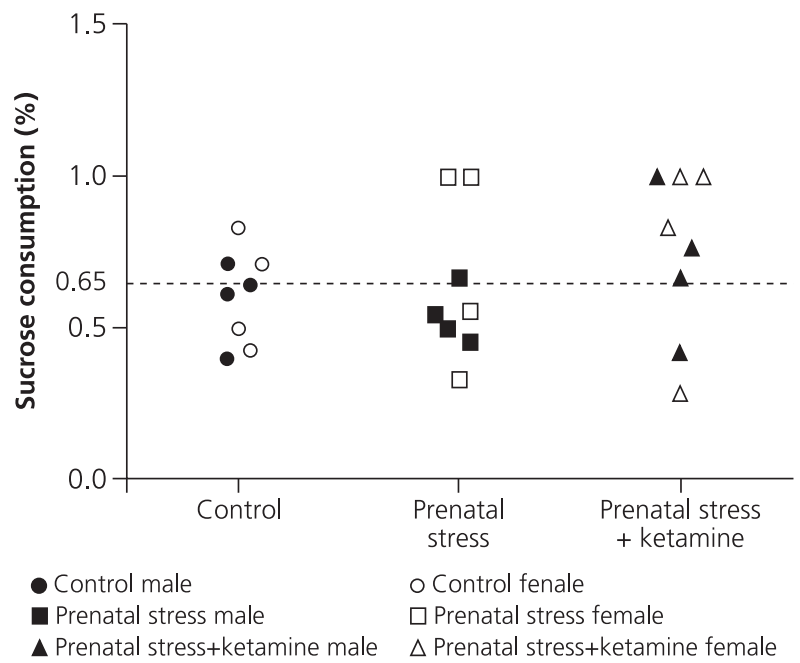

Figure 2. The sucrose preference test results of control, saline or ketamine administered prenatally stressed rats ( $n=8$ animals for each group). Data shows the sucrose consumption ratio of each animal with white symbols indicating female and black symbols indicating male groups. Dashed line shows the threshold level (0.65\%) of the anhedonic response.

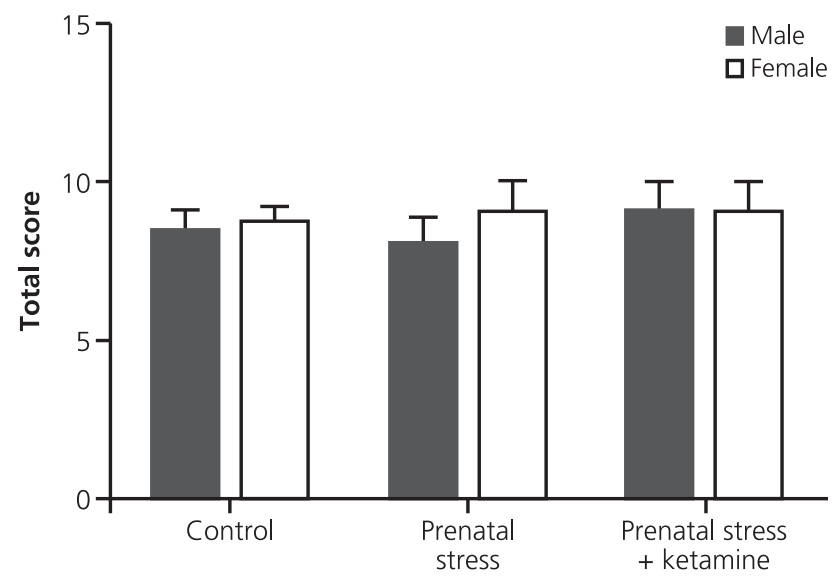

Figure 3. The modified grip suspension test results of control, saline or ketamine administered prenatally stressed rats ( $n=8$ animals for each group) displayed no significant differences among groups. Data shows the average scores \pm SEM of each group for male (black bars) and female (white bars) animals. 
ilar immobilization time across groups no significant difference between control and stress groups $(38.13 \pm 17.1$ in controls, $45.01 \pm 20.9$ in prenatal stress, and $46.25 \pm 21.0$ in prenatally stressed and ketamine treated group). However, in males, prenatal stress exposure increased the immobility duration from $27.88 \pm 20.8$ to $60.01 \pm 24.2$ seconds, in comparison to control animals (Figure 4). Immobility time of male animals displayed a reduction $(21.00 \pm 24.2$ seconds $)$ in the forced swim test following ketamine treatment.

After completion of behavioral tests, we examined stress-induced immediate gene expression by using c-Fos immunohistochemistry. The representative sections from the region of interest were shown in Figure 5 and the outline of the medial prefrontal cortical region is indicated in the middle section. Within this region, approximately 8-10 sampling sites were defined by systematic random sampling method, and c-Fos expressing nuclear profiles was counted to estimate the neuronal activity levels (Figure 5a). Immediate gene expression level was different in control and experimental groups as estimated by quantitative analyses (Figure 5b). Two-way ANOVA results displayed a significant group $[\mathrm{F}(2,18)=8.47, \mathrm{p}=0.0026]$ and gender difference $[\mathrm{F}(1,18)=25.71, \mathrm{p}<0.0001]$. There was also a significant group-gender interaction $[(\mathrm{F} 2,18=4.69)$, $\mathrm{p}=0.0229]$. These differences were due to the enhanced cFos expression in prenatally stressed females compared to control groups, and ketamine treatment decreased neuronal activation similar to control levels. In ketamine treated group, there is also a significant gender difference. On the other hand, there was no significant difference among male groups.

a

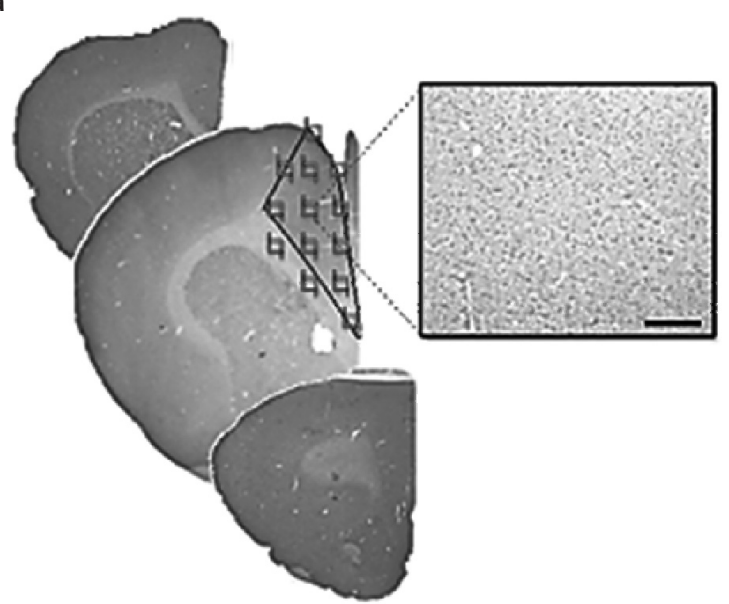

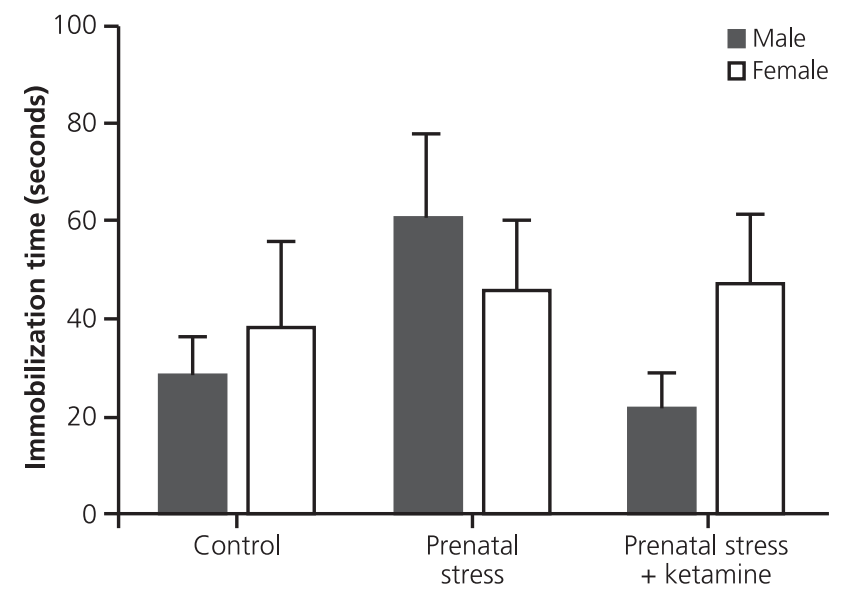

Figure 4. The forced swim test results of control, saline or ketamine administered prenatally stressed rats ( $n=8$ animals for each group) displayed no significant differences among groups. Data shows the mean immobilization duration \pm SEM of each group for male (black bars) and female (white bars) animals.

\section{Discussion}

In this study, we have investigated the effect of single-dose ketamine application in prenatally stressed juvenile rats. Although stress protocol used in this study did not significantly alter the active coping and depressive-like behavior of pups, ketamine application caused alterations in some behavioral parameters and immediate gene expression in the prefrontal cortical region were observed in prenatally stressed animals. In previous studies, the antidepressantlike effect of single or repeated dose of ketamine treat-

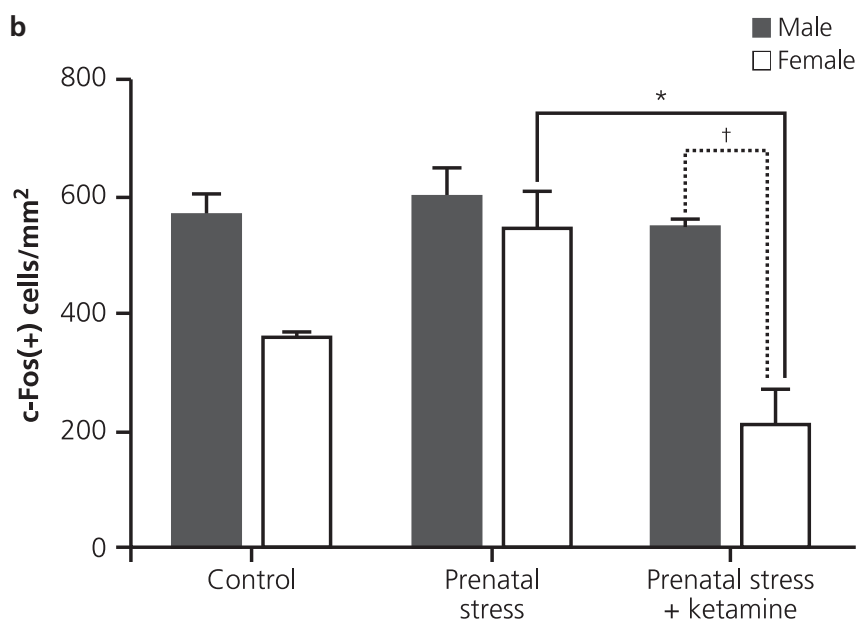

Figure 5. The estimation of neuronal density of c-Fos (+) neurons in the medial PFC outlined with black lines in (a) was done by using systematic random quantification method. Quantitative analyses showed significant ( $\left.{ }^{*} p<0.001\right)$ differences between prenatally stressed and ketamine treated groups in females $(\mathbf{b})$. There was also a significant gender difference $\left({ }^{\dagger} p<0.01\right)$ in ketamine administered group. Data shows the mean \pm SEM of the number of c-Fos expressing neurons per unit area. Scale bar $=250 \mu \mathrm{m}$. 
ment has been investigated in normal or chronically stressed animals, but none of the experimental studies examined its effects in prenatally stressed rodents. A general impairment of the hypothalamo-pituitary-adrenal (HPA) axis is characteristic for prenatally stressed animals and the prolonged corticosterone secretion in response to stress was positively correlated with alterations in behavioral tests, such as sucrose preference or FST. ${ }^{[29,30]}$ Therefore, prenatal stress exposure model has a face validity with some clinical features observed especially in juvenile depression patients.

In rodents, exposure to stressors in an unpredictable sequence over a prolonged time period satisfies most of the criteria of validity as an animal model of depression. ${ }^{[31]}$ By using this model, it has been shown that acute or chronic treatment with ketamine reverses the increase in adrenal gland weight, normalizes circulating corticosterone and ACTH levels and promotes regain of body weight. ${ }^{[32]}$ Although similar physiological alterations have also been shown by other investigators, in these studies, acute ketamine treatment failed to reverse anhedonic response in rats exposed to chronic mild stress. ${ }^{[33]}$ These results suggest that stress protocols might differentially influence the behavioral outcomes of ketamine treatment.

In experimental studies, anhedonia is widely measured by sucrose preference test and reduced sucrose consumption corresponds to a depressive-like symptom. ${ }^{[34]}$ Chronic unpredictable stress exposure for 3 weeks significantly decreases the preference for sweetened solution and increases the latency in novelty suppressed feeding test, and these effects are reversed by single-dose ketamine treatment. ${ }^{[2]}$ In our study, the number of prenatally stressed animals passing the threshold level of the sucrose consumption ratio showed an increase following ketamine treatment. Similarly, Garcia et al ${ }^{[32]}$ showed that in rats exposed to chronic stress, repeated administration of ketamine (7 days) reversed the decrease in sucrose consumption. In another study, administration of a low dose of ketamine $(0.5 \mathrm{mg} / \mathrm{kg})$ for 10 days significantly increased sucrose consumption in Wistar-Kyoto rats. ${ }^{[35]}$ Furthermore, Li et al ${ }^{[22]}$ reported that even a single-dose of ketamine produces a long lasting (up to 7 days) increase in sucrose preference relative to chronic unpredictable stress exposed animals. Protracted effects of acute ketamine treatment were also evident in mice exposed to chronic mild stress that is tested at 4,6 , and 8 days after a single ketamine treatment. ${ }^{[36]}$ These data suggest that sucrose preference of animals might be used as a sensitive screening test for rapid-acting antidepressant drugs like ketamine.

In addition to rewarding behaviors, the effects of ketamine have also been shown on aversive circumstances, such as elevated plus-maze or FST. ${ }^{[21,32,37-39]}$ While elevated plus-maze is frequently used to measure anxiety behavior in rodents, FST is the most frequently used behavioral despair test for measuring depressive-like behavior, based on an immobility response induced by inescapable exposure to stress. ${ }^{[40,4]}$ FST has strong predictive validity because short-term administration of antidepressant compounds from a variety of pharmacological classes reduces immobility time in FST. ${ }^{[42]}$ The most commonly prescribed antidepressants such as tricyclic anti-depressants and selective serotonin reuptake inhibitors (SSRIs) have significant limitations with delayed onset of action and their effects do not last beyond a few hours following their acute administration. ${ }^{[43]}$ Whereas, effects of ketamine are distinct from those conventional drugs since it produces protracted behavioral effects persisting from one to several days after administration. ${ }^{[2]}$ The majority of studies indicate that the FST remains sensitive to the protracted effects of ketamine up to 1 week after a single injection. ${ }^{[4+50]}$ Different groups were reported diverse durations persisting for 8 days, ${ }^{[3]} 10$ days, ${ }^{[51]} 12$ days, ${ }^{[52]}$ and 2 weeks. ${ }^{[53]}$ Interestingly, repeated ketamine application (20 $\mathrm{mg} / \mathrm{kg}$ ) for 15 days has been shown to have anxiolytic- and antidepressant-like responses even 2 months after the cessation of treatment in adolescent rats. ${ }^{[5]}$ These results are in agreement with other studies utilizing a 10- or 12-day dosing regimen to establish longer-lasting effects of chronic ketamine on depressive-like activity in the FST ${ }^{[5,56]}$ In the literature, there are significant dose, gender and strain-dependent differences in the sensitivity to ketamine treatment. For example, Wistar rats are insensitive to the antidepressant-like effects of low dose ketamine (2.5 and $5 \mathrm{mg} / \mathrm{kg}$ ) in comparison to Wistar-Kyoto rats. ${ }^{[55]}$ Interestingly, in majority of studies, behavioral consequences of ketamine treatment have been investigated in adult male rats. ${ }^{[3,46-49]}$ In our study, the effects of ketamine were investigated in both females and males, but more prominent behavioral alterations were observed in males. In the literature, there is a clear consensus that efficacy of antidepressant drugs show gender-dependent variations. In one study comparing the behavioral and molecular effects of ketamine in both male and female rats, it has been demonstrated that low dose of ketamine does not have antidepressant-like effects in males. ${ }^{[4]}$ In contrast, females have a greater sensitivity to lower doses of ketamine, and these effects might be completely abolished when female rats were ovariectomized and then restored when physiological levels of estrogen and progesterone were supplemented. These results suggest that gonadal hormones play a critical role in determining the effectiveness of ketamine treatment in rats. Likewise, in mice subjected to chronic mild stress, a single-dose of ketamine 
injection $(10 \mathrm{mg} / \mathrm{kg})$ can induce sex-dependent behavioral effects for five weeks. Although female mice were shown more reactive to the earlier effects of ketamine, the antidepressant potential of this drug was proved to last longer in male animals. ${ }^{[5]}$ A recent clinical study suggested that sex differences in glutamate receptor gene expression might also play a major role in depression and antidepressant efficacy of drugs. ${ }^{[3]}$ In a large cohort of postmortem subjects from MDD patients and controls, more generalized and severe disruption in the regulation of the glutamate receptors were found in females, but more specific alterations were reported in males.

Prenatal stress exposure not only leads to long-term behavioral consequences, but also causes neurobiological and morphological alterations in the offspring. ${ }^{[2,57]}$ Functional imaging studies revealed that stress experience dramatically decreased brain activity especially in the regions associated with the limbic system. ${ }^{[58]}$ Within this system, PFC has a particular importance, since it receives input from all other cortical areas and modulates motor, cognitive, affective and social behavior across time. Moreover, prolonged developmental pace makes this neocortical region more vulnerable to both developmental and environmental insults, which is often manifested in neuropsychiatric disorders. ${ }^{[59]}$ In a current study, we evaluated the morphological changes in the medial $\mathrm{PFC}$ of rats subjected to perinatal stress exposure. The dendritic complexity and spine density of pyramidal neurons located in this region displayed an anxiety level- and sex-dependent reduction, especially in the stressed males. ${ }^{[16]}$ Moreover, total number estimation of c-Fos (+) nuclei, indicating the neuronal activation upon stressful challenge, significantly increased in high anxious animals compared with low anxious and control groups, in both gender. These results suggest that medial PFC is a critical site of neural plasticity within the stressor controllability paradigm. Since morphological outcomes of early life stress show correlation with the behavior of animals, in the present study, to investigate the efficacy of ketamine treatment, we compared the immediate gene expression of neurons in the medial PFC between control and experimental groups. Our results showed that density of neuronal activation, estimated by using c-Fos immunohistochemistry, have significant gender- and group- dependent differences. However, in contrast to behavioral outcomes, alterations in the immediate gene expression were more prominent in females than those of males. We thought that these differences were high likely due to the diverse stimulation of reward circuitry in female and male animals. In a recent study, Chang et al. ${ }^{[60]}$ showed that activation of neurons in the ventrome- dial PFC and projections from this region to the nucleus accumbens play a key role in regulation of reward-related processes and actions of diverse antidepressant drugs. Therefore, it is possible that in prenatally stressed female animals, enhanced activation of neurons in the medial PFC might cause an increase in the sucrose consumption of animals by stimulating the neurons located in the nucleus accumbens. In contrast, sustained activation of this pathway might contribute to the anhedonic response observed in prenatally stressed males.

Neuroimaging studies in humans supports these ideas by demonstrating that ketamine increases the activity of PFC. ${ }^{[60,61]}$ Likewise, rodent studies showed that ketamine treatment causes rapid increases of glutamate transmission in PFC ${ }^{[2]}$ It is high likely that ketamine's actions on synapses might reverse the morphological and functional alterations in the medial PFC neurons caused by stress exposure. In a recent study, using optogenetic stimulation of infralimbic PFC, Fuckikami et al. ${ }^{[63]}$ showed that rapid and long-lasting antidepressant and anxiolytic effects of systemic ketamine administration is associated with increased number and function of spine synapses of layer $\mathrm{V}$ pyramidal neurons of PFC. Therefore, in the future studies, we are planning to assess the impact of ketamine application on neuronal plasticity by investigating the dendritic morphology of neurons located in different limbic regions.

\section{Conclusion}

Both clinical and animal studies report that subanesthetic dose of ketamine administration produces antidepressantlike response, especially in treatment-resistant depression cases. Ketamine's rapid and sustained actions have been investigated in normal or chronically stressed animals, but none of the experimental studies has examined its effects in prenatally stressed rats. Using a combination of behavioral tests and c-Fos immunohistochemistry, we demonstrated that a single-dose ketamine treatment produces antidepressant-like effect in juvenile male rats and enhances the neuronal activation in the medial prefrontal cortical region in female rats exposed to prenatal stress.

\section{References}

1. World Health Organization. The world health report 2001 - Mental Health: new understanding, new hope. Geneva: WHO; 2001.

2. Andrade L, Caraveo-A. Epidemiology of major depressive episodes: Results from the international consortium of psychiatric epidemiology (ICPE) surveys. Int J Methods Psychiatr Res 2003; $12: 3-21$.

3. Gray AL, Hyde TM, Deep-Soboslay A, Kleinman JE, Sodhi MS. Sex differences in glutamate receptor gene expression in major depression and suicide. MS Mol Psychiatry 2015;20:1057-68. 
4. Katz MM, Tekell JL, Bowden CL, Brannan S. Houston JP, Bermann N, Frazer A. Onset and early behavioral effects of pharmacologically different antidepressants and placebo in depression. Neuropsychopharmacology 2004;29:566-79.

5. Gagné GG Jr, Furman MJ, Carpenter LL, Price LH. Efficacy of continuation ECT and antidepressant drugs compared to longterm antidepressants alone in depressed patients. Am J Psychiatry 2000;157:1960-5

6. Duman RS, Li N, Liu RJ, Duric V, Aghajanian G. Signaling pathways underlying the rapid antidepressant actions of ketamine. Neuropharmacology 2012;62:35-41.

7. $\mathrm{M}$ aan het Rot, CA Zarate Jr, DS Charney, SJ. Ketamine for depression: where do we go from here? Biol Psychiatry 2012;72: 537-47.

8. Murrough JW, Losifescu VD, Chang LC. antidepressant efficacy of ketamine in treatment-resistant major depression: a two-site randomized controlled trial. Am J Psychiatry 2013; 170:1134-42.

9. Price BR, Losifescu VD, Murrough JW. Effects of ketamine on explicit and implicit suicidal cognition: a randomized controlled trial in treatment-resistant depression. Depress Anxiety 2014;31: $335-43$.

10. Romeo B, Choucha W, Fossati P, Rotge JY. Meta-analysis of short- and mid-term efficacy of ketamine in unipolar and bipolar depression. Psychiatry Res 2015;230:682-88.

11. Li N, Liu RJ, Dwyer JM, Banasr M, Lee B, Son H, Li XY, Aghajanian G, Duman RS. Glutamate NMDA receptor antagonists rapidly reverse behavioral and synaptic deficits caused by chronic stress exposure. Biol Psychiatry 2011;69:754-61.

12. Heller AS, Johnstone T, Peterson MJ, Kolden GG, Kalin NH, Davidson RJ. Increased prefrontal cortex activity during negative emotion regulation as a predictor of depression symptom severity trajectory over 6 months. JAMA Psychiatry 2013;70:1181-9.

13. Kang HJ, Voleti B, Hajszan T, Rajkowska G, Stockmeier CA, Licznerski P, Lepack A, Majik MS, Jeong LS, Banasr M, Son H, Duman RS. Decreased expression of synapse-related genes and loss of synapses in major depressive disorder. Nat Med 2012;18:1413-7.

14. Grimm S, Luborzewski A, Schubert F, Merkl A, Kronenberg G, Colla M, Heuser I, Bajbouj M. Region-specific glutamate changes in patients with unipolar depression. J Psychiatr Res 2012;46: 1059-65.

15. Muhammad A, Kolb B. Mild prenatal stress-modulated behavior and neuronal spine density without affecting amphetamine sensitization. Dev Neurosci 2011;33:85-98.

16. Soztutar E, Colak E, Ulupinar E. Gender- and anxiety leveldependent effects of perinatal stress exposure on medial prefrontal cortex. Exp Neurol 2015;2:274-84.

17. Li N, Liu RJ, Dwyer JM, Duman RS. Glutamate NMDA receptor antagonists rapidly reverse behavioral and synaptic deficits caused by chronic stress exposure. Biol Psychiatry 2011;69:754-61.

18. Långsjö JW, Salmi E, Kaisti KK, Aalto S, Hinkka S, Aantaa R, Oikonen V, Viljanen T, Kurki T, Silvanto M, Scheinin H. Effects of subanesthetic ketamine on regional cerebral glucose metabolism in humans. Anesthesiology 2004;100:1065-71.

19. Murrough JW. Ketamine as a novel antidepressant: from synapse to behavior. Clin Pharmacol Ther 2012;91:303-9.

20. Li N, Lee B, Liu RJ, Banasr M, Dwyer JM, Iwata M, Li XY, Aghajanian G, Duman RS. mTOR-dependent synapse formation underlies the rapid antidepressant effects of NMDA antagonists. Science 2010;329:959-64.

21. Autry AE, Adachi M, Nosyreva E, Na ES, Los MF, Cheng PF, Kavalali ET, Monteggia LM. NMDA receptor blockade at rest triggers rapid behavioural antidepressant responses. Nature 2011;475:91-5.

22. Li N, Liu RJ, Dwyer JM, Banasr M, Lee B, Son H, Li XY, Aghajanian G, Duman RS. Glutamate N-methyl-D-aspartate receptor antagonists rapidly reverse behavioral and synaptic deficits caused by chronic stress exposure. Biol Psychiatry 2011;69:754-61.

23. Willner P. Validity, reliability and utility of the chronic mild stress model of depression: a 10-year review and evaluation. Psychopharmacology (Berl) 1997;134:319-29.

24. Ulupinar E, Erol K, Ay H, Yucel F. Rearing conditions differently affect the motor performance and cerebellar morphology of prenatally stressed juvenile rats. Behav Brain Res 2015;278:235-43.

25. Carrier N, Kabbaj M. Sex differences in the antidepressant like effects of ketamine. Neuropharmacology 2013;70:27-34.

26. Paxinos G, Watson C. The rat brain in stereotaxic coordinates. San Diego (CA): Academic Press; 1986.

27. Weinstock $M$. The long-term behavioural consequences of prenatal stress. Neurosci Biobehav Rev 2008;32:1073-86.

28. Porsolt RD, Anton G, Blavet N, Jalfre M. Behavioural despair in rats: a new model sensitive to antidepressant treatments. Eur J Pharmacol 1978;47:379-91.

29. Morley-Fletcher S, Darnaudery M, Koehl M, Casolini P, Van Reeth O, Maccari S. Prenatal stress in rats predicts immobility behavior in the forced swim test. Effects of a chronic treatment with tianeptine. Brain Res 2003;989:246-51.

30. Weinstock M. Sex-dependent changes induced by prenatal stress in cortical and hippocampal morphology and behaviour in rats: an update. Stress 2011;14:604-13.

31. Willner P. Chronic mild stress (CMS) revisited: consistency and behavioural-neurobiological concordance in the effects of CMS. Neuropsychobiology 2005;52:90-110.

32. Garcia LSB, Comim CM, Valvassori SS, Réus GZ, Stertz L, Kapczinski F, Gavioli EC, Quevedo J. Ketamine treatment reverses behavioral and physiological alterations induced by chronic mild stress in rats. Prog Neuropsychopharmacol Biol Psychiatry 2009;33:450-55.

33. Rezin GT, Goncalves CL, Daufenbach JF, Fraga DB, Santos PM, Ferreira GK, Hermani FV, Comim CM, Quevedo J, Streck EL. Acute administration of ketamine reverses the inhibition of mitochondrial respiratory chain induced by chronic mild stress. Brain Res Bull 2009;79:418-21.

34. Browne CA, Lucki I. Antidepressant effects of ketamine: mechanisms underlying fast-acting novel antidepressants. Front Pharmacol 2013;4:161.

35. Akinfiresoye L, Tizabi Y. Antidepressant effects of AMPA and ketamine combination: role of hippocampal BDNF, synapsin, and mTOR. Psychopharmacology 2013;230:291-8.

36. Ma XC, Dang YH, Jia M, Ma R, Wang F, Wu J, Gao CG, Hashimoto K. Long-lasting antidepressant action of ketamine, but not glycogen synthase kinase-3 inhibitor SB216763, in the chronic mild stress model of mice. PLoS ONE 2013;8:e56053.

37. Maeng S, Zarate CA Jr, Du J, Schloesser RJ, McCammon J, Chen G, Manji HK. Cellular mechanisms underlying the antidepressant effects of ketamine: role of alpha-amino-3-hydroxy-5-methylisoxazole-4-propionic acid receptors. Biol Psychiatry 2008;63:349-52. 
38. Popik P, Kos T, Sowa-Kucma M, Nowak G. Lack of persistent effects of ketamine in rodent models of depression. Psychopharmacology 2008;198:421-30.

39. Engin E, Treit D, Dickson CT, Anxiolytic- and antidepressantlike properties of ketamine in behavioral and neurophysiological animal models. Neuroscience 2009;161:359-69.

40. Bourin, M. Animal models of anxiety: are they suitable for predicting drug action in humans? Polish J Pharmacol1997;49:79-84.

41. Can ÖD, Ulupınar E, Özkay ÜD, Yegin B, Öztürk Y. The effect of simvastatin treatment on behavioral parameters, cognitive performance, and hippocampal morphology in rats fed a standard or a high-fat diet. Behav Pharmacol 2012;23:582-92.

42. Cryan JF, Valentino RJ, Lucki I. Assessing substrates underlying the behavioral effects of antidepressants using the modified rat forced swimming test. Neurosci Biobehav Rev 2005;29:547-69.

43. Hoshaw BA, Hill TI, Crowley JJ, Malberg JE, Khawaja X, Rosenzweig LS, Schechter LE, Lucki I. Antidepressant-like behavioral effects of IGF-I produced by enhanced serotonin transmission. Eur J Pharmacol 2008;594:109-16.

44. Burgdorf J, Zhang XL, Nicholson KL, Balster RL, Leander JD, Stanton PK, Gross AL, Kroes RA, Moskal JR. GLYX-13, a NMDA receptor glycine-site functional partial agonist, induces antidepressant-like effects without ketamine-like side effects. Neuropsychopharmacology 2013;38:729-42.

45. Carrier N, Kabbaj M. Sex differences in the antidepressant like effects of ketamine. Neuropharmacology 2013;70:27-34.

46. Gigliucci V, O’Dowd G, Casey S, Egan D, Gibney S, Harkin A. Ketamine elicits sustained antidepressant-like activity via a serotonin-dependent mechanism. Psychopharmacology 2013;228:15766.

47. Koike H, Iijima M, Chaki S. Effects of ketamine and LY341495 on the depressive-like behavior of repeated corticosterone-injected rats. Pharmacol Biochem Behav 2013;107:20-3.

48. Koike H, Fukumoto K, Iijima M, Chaki S. Role of BDNF/TrkB signaling in antidepressant-like effects of a group II metabotropic glutamate receptor antagonist in animal models of depression. Behav Brain Res 2013;238:48-52.

49. Muller HK, Wegener G, Liebenberg N, Zarate, CA Jr, Popoli M, Elfving B. Ketamine regulates the presynaptic release machinery in the hippocampus. J Psychiatr Res 2013;47:892-9.

50. Walker AK, Budac DP, Bisulco S, Lee AW, Smith RA, Beenders B, Kelley KW, Dantzer R. NMDA receptor blockade by ketamine abrogates lipopolysaccharide-induced depressive-like behavior in C57BL/6J Mice. Neuropsychopharmacology 2013;38:1609-16.

51. Yilmaz A, Schulz D, Aksoy A, Canbeyli R. Prolonged effect of an anesthetic dose of ketamine on behavioral despair. Pharmacol Biochem Behav 2002;71:341-4.
52. Garcia LS, Comim CM, Valvassori SS, Reus GZ, Barbosa LM, Andreazza AC, Stertz L, Fries GR, Gavioli EC, Kapczinski F, Quevedo J. Acute administration of ketamine induces antidepressant-like effects in the forced swimming test a decreases BDNF levels in the rat hippocampus. Prog Neuropsychopharmacol Biol Psychiatry 2008;32:140-4.

53. Maeng S, Zarate CA Jr, Du J, Schloesser RJ, McCammon J, Chen G, Manji HK. Cellular mechanisms underlying the antidepressant effects of ketamine: role of alpha-amino-3-hydroxy-5-methylisoxazole-4-propionic acid receptors. Biol Psychiatry 2008;63:349-52.

54. Parise EM, Alcantara LF, Warren BL, Wright KN, Hadad R, Sial OK, Kroeck KG, Iñiguez SD, Bolaños-Guzmán CA. Repeated ketamine exposure induces an enduring resilient phenotype in adolescent and adult rats. Biol Psychiatry 2013;74:750-9.

55. Tizabi Y, Bhatti BH, Manaye KF, Das JR, Akinfiresoye L. Antidepressant-like effects of low ketamine dose is associated with increased hippocampal AMPA/NMDA receptor density ratio in female Wistar-Kyoto rats. Neuroscience 2012;213:72-80.

56. Franceschelli A, Sens J, Herchick S, Thelen C, Pitychoutis PM. Sex differences in the rapid and the sustained antidepressant-like effects of ketamine in stress-naïve and "depressed" mice exposed to chronic mild stress. Neuroscience 2015;290:49-60.

57. Ulupinar E, Yucel F, Ortug G. The effects of prenatal stress on the Purkinje cell neurogenesis. Neurotoxicol Teratol 2006;28:86-94.

58. Bock J, Riedel A, Braun K. Differential changes of metabolic brain activity and interregional functional coupling in prefronto-limbic pathways during different stress conditions: functional imaging in freely behaving rodent pups. Front Cell Neurosci 2012;10:6-19.

59. Kolb B, Mychasiuk R, Muhammad A, Li Y, Frost DO, Gibb R. Experience and the developing prefrontal cortex. Proc Natl Acad Sci U S A 2012;109:S17186-93.

60. Chang $\mathrm{CH}$, Chen MC, Lu J. Effect of antidepressant drugs on the vmPFC-limbic circuitry. Neuropharmacology 2015;92:116-24.

61. Långsjö JW, Kaisti KK, Aalto S, Hinkka S, Aantaa R, Oikonen V, Sipilä H, Kurki T, Silvanto M, Scheinin H. Effects of subanesthetic doses of ketamine on regional cerebral blood flow, oxygen consumption, and blood volume in humans. Anesthesiology 2003;99:614-23.

62. Holcomb HH, Lahti AC, Medoff DR, Cullen T, Tamminga CA. Effects of noncompetitive NMDA receptor blockade on anterior cingulate cerebral blood flow in volunteers with schizophrenia. Neuropsychopharmacology 2005;30:2275-82.

63. Fuchikami M, Thomas A, Liu R, Wohleb ES, Land BB, DiLeone RJ, Aghajanian GK, Duman RS. Optogenetic stimulation of infralimbic PFC reproduces ketamine's rapid and sustained antidepressant actions. Proc Natl Acad Sci U S A 2015;112:8106-11.

This is an open access article distributed under the terms of the Creative Commons Attribution-NonCommercial-NoDerivs 3.0 Unported (CC BY-NCND3.0) Licence (http://creativecommons.org/licenses/by-nc-nd/3.0/) which permits unrestricted noncommercial use, distribution, and reproduction in any medium, provided the original work is properly cited. Please cite this article as: Polat Çorumlu E, Aydın OÖ, Aydın EG, Ulupınar E. Effects of single-dose ketamine infusion on behavioral parameters and neuronal activation in the medial prefrontal cortex of juvenile rats exposed to prenatal stress. Anatomy $2015 ; 9(3): 142-150$.

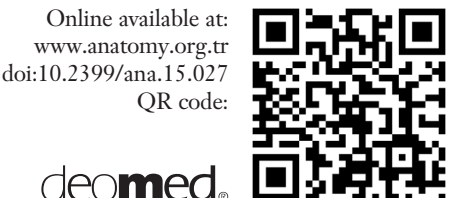

Correspondence to: Emel Ulupınar, MD, PhD

Department of Anatomy, Faculty of Medicine,

Eskişehir Osmangazi University, 26040, Eskişehir, Turkey

Phone: +90222 $2392979 / 4436$

e-mail: eulupi@ogu.edu.tr

Conflict of interest statement: No conflicts declared. 